\title{
Une forme de tératozoospermie monomorphe : Le syndrome des spermatozoïdes macrocéphales et ses variants
}

\author{
Catherine METZLER-GUILLEMAIN1, Vincent ACHARD1, Georges MERCIER ${ }^{2}$, \\ Marie-Roberte GUICHAOUA ${ }^{1}$
}

Catherine METZLER-GUILLEMAIN1, Vincent ACHARD1, Georges MERCIER2, Marie-Roberte GUICHAOUA1

\section{RESUME}

Les tératozoospermies monomorphes reflètent une anomalie de structure des spermatozoïdes rare, mais sont souvent source de discussion lorsqu'il faut envisager une prise en charge thérapeutique. Nous proposons donc de faire le point des données actuelles de la littérature concernant le syndrome des spermatozoïdes macrocéphales et ses variants.

D'un point de vue pratique, en dehors de la forme homogène du syndrome, l'assistance médicale à la procréation (AMP) peut être proposée. Après évaluation des taux d'anomalies chromosomiques dans les spermatozoïdes de ces patients, une discussion multidisciplinaire et une information claire du couple sont nécessaires avant toute prise en charge en AMP.

Mots clés : tératozoospermie, spermatozoïdes macrocéphales

\section{INTRODUCTION}

Une tératozoospermie est dite monomorphe, lorsque que la totalité ou la majorité des spermatozoïdes anormaux présentent la ou les mêmes anomalies. Ces anomalies morphologiques qui reflètent une anomalie de structure des spermatozoïdes sont rares, mais sont souvent source de discussion lorsqu'il faut envisager une prise en charge thérapeutique en assistance médicale à la procréation (AMP). Parmi les tératospermies monomorphes les plus fréquemment retrouvées, on décrit les spermatozoïdes décapités, les globozoospermies, les spermatozoïdes à flagelle court et les spermatozoïdes macrocéphales. Les premières sont décrites en détails dans l'article consacré à l'altération des spermatozoïdes en microscopie électronique [7].

Nous proposons ici de faire le point des données actuelles de la littérature concernant les spermatozoïdes macrocéphales, notamment en cas de forme atypique, et de proposer une conduite à tenir thérapeutique.

\section{FORME CLASSIQUE ET VARIANTS PHÉNOTYPIQUES}

La forme typique du syndrome des spermatozoïdes macrocéphales polyflagelles est caractérisée par une augmentation du volume nucléaire qui peut atteindre 4 fois le volume d'un noyau de spermatozoïde normal, une tête et un acrosome très irréguliers et la présence de 4 flagelles. Quelques spermatozoïdes macrocéphales se présentent sous la forme de spermatozoïdes dupliqués contenant deux noyaux étroitement apposés. A côté de la forme classique étiquetée type 1, Escalier décrit un type 2 avec arrêt au cours de la spermiogenèse et un type 3 dans lequel les spermatozoïdes macrocéphales présentent un arrêt de la croissance de l'axonème et des anomalies périaxonémales [6].

Ce phénotype s'associe à une infertilité sévère liée aux anomalies morphologiques que nous venons de décrire, à une oligoasthénozoospermie et à la présence d'un déséquilibre chromosomique dans la plupart des spermatozoïdes analysés par hybridation in situ fluorescente (FISH). Ce syndrome est classiquement décrit comme résultant d'un défaut de division

\section{Correspondance :}

Dr Catherine METZLER-GUILLEMAIN - Laboratoire de Biologie de la Reproduction, Hôpital de la Conception, 147 Boulevard Baille, 13585, Marseille Cedex 5, France -

Email catherine.guillemain@ap-hm.fr 
méiotique, mais il est probable qu'une réduction de l'activité mitotique spermatogoniale participe à la constitution de l'oligozoospermie constante chez ces patients [1]. L'arrêt du développement d'un nombre plus ou moins important de spermatocytes et de spermatides macrocéphales représente une autre cause de l'oligozoospermie.

A côté des formes typiques que nous venons de décrire dans lesquelles $100 \%$ ou presque des spermatozoïdes sont de grande taille, nous avons mis en évidence des formes partielles où coexistent des spermatozoïdes de taille normale et des spermatozoïdes macrocéphales [16]. Deux observations avec respectivement $64 \%$ et $54 \%$ de spermatozoïdes macrocéphales ont été publiées ensuite $[13,14]$, puis nous avons à nouveau décrit quatre patients dont les taux étaient respectivement de $19 \%$, $22 \%, 29,5 \%$ et $49,7 \%$ [1].

Dans ces formes, l'analyse automatisée de la surface des spermatozoïdes révèle soit l'existence de 2 populations de spermatozoïdes, l'une normale, l'autre correspondant au clone macrocéphale, soit l'existence de toutes les tailles intermédiaires entre les spermatozoïdes normaux et les spermatozoïdes macrocéphales [1]. Ces quatre patients infertiles présentaient tous une oligoasthénozoopermie, chez deux d'entre eux le spermocytogramme montrait aussi la présence d'une tératozoospermie polymorphe, tandis que l'interrogatoire révélait l'existence de cinq morts périnatales dans la fratrie de l'un de ces patients.

\section{PHYSIOPATHOLOGIE}

S'il est maintenant acquis que ce syndrome résulte d'une absence de cytokinèse et de cytodiérèse, le mécanisme impliqué dans l'apparition de cette symptomatologie est mal connu. Dans le spermatocyte au stade pachytène, l'appareil de Golgi ne se divise pas et ne migre pas aux pôles opposés de la cellule comme lors de la prophase méiotique normale [6]. Bien que la membrane nucléaire disparaisse, les centrosomes ne se séparent pas, le fuseau méiotique bipolaire ne se constitue pas ou est très court, et la cellule ne progresse jamais vers la métaphase, l'anaphase et la télophase sont absentes. Un certain nombre de spermatocytes échappent aux points de contrôles tels que le point de contrôle du pachytène ou celui contrôlant l'assemblage du fuseau, et vont donner naissance à des spermatides géantes puis des spermatozoïdes macrocéphales. II est surprenant de voir que, excepté le caractère unilatéral de la manchette, les autres événements de la spermiogenèse se déroulent normalement. D'autres spermatocytes sont arrêtés par ces points de contrôle et dégénèrent, participant ainsi à l'oligospermie associée à ce syndrome.

La FISH sur les spermatozoïdes macrocéphales révèle la présence d'anomalies de nombre des chromosomes, polyploïdies ou aneuploïdies complexes $[1,4,8,14,16]$. La polyploïdie se manifeste par la présence de spermatozoïdes le plus souvent diploïdes, moins fréquemment tétraploïdes et exceptionnellement triploïdes. Ces diverses polyploïdies résultent de l'absence de l'une ou des deux divisions méiotiques. Quant à l'aneuploïdie, elle peut être expliquée par le fait qu'un certain nombre de spermatocytes sont capables de réaliser l'une ou les deux divisions méiotiques, mais les fuseaux de division n'étant que partiellement fonctionnels, il se produit des non-disjonctions chromosomiques sur un nombre plus ou moins important de bivalents à la première division et de chromosomes à la deuxième division. De plus, la fixation des chromosomes aux pôles du fuseau peut être défectueuse en raison de jeux incomplets de kinétochores au niveau des centromères. L'orientation anormale des centromères sur la plaque métaphasique est inadaptée à une ségrégation équitable des chromosomes lors des anaphases I et II. Ce type d'anomalies active le point de contrôle d'assemblage du fuseau qui éliminerait encore un certain nombre de spermatocytes.

Ces différents mécanismes expliquent la très grande variété d'anomalies chromosomiques que l'on peut observer dans les spermatozoïdes de ces patients [1, 4].

L'origine génétique du syndrome des spermatozoïdes macrocéphales polyflagelles classique est fortement suggérée par la présence de l'anomalie dans $100 \%$ des spermatozoïdes chez un certain nombre de patients, de l'existence de cas familiaux et de la présence de morts périnatales dans la fratrie de deux sujets atteints [1, 2]. L'existence de ces morts périnatales suggère qu'il puisse y avoir une atteinte concomitante des cycles mitotiques et méiotiques par une mutation d'un gène s'exprimant dans les cellules somatiques et germinales.

Cette mutation pourrait être dominante avec une expressivité variable et une pénétrance incomplète [2]. Plusieurs gènes sont potentiellement candidats pour ces phénotypes $[2,6]$. Une mutation d'un gène (AURKC) a été récemment identifiée chez dix patients porteurs du syndrome des spermatozoïdes macrocéphales. Il s'agit d'une délétion d'un seul nucléotide dans une séquence codante [5] qui aboutit à la formation d'une protéine tronquée dépourvue du domaine kinase.

II n'en est pas de même des formes partielles de ce syndrome, dont l'origine génétique n'est pas évidente. Plusieurs hypothèses peuvent être discutées pour tenter d'expliquer la coexistence des formes typiques et des formes macrocéphales dans un même éjaculat.

L'explication la plus simple est qu'il existerait une mosaïque germinale résultant de la survenue d'une mutation spontanée survenant soit dans une cellule germinale qui continue à se diviser, soit plus précocement, avant la détermination des cellules germinales [17]. Dans ce cas, des cellules germinales et somatiques peuvent être affectées dans leur cycle cellulaire.

Une autre explication est qu'une mutation ne s'exprimerait que lorsque la protéine est produite en quantité insuffisante, en deçà d'un seuil, dans un environnement testiculaire défectueux.

Enfin, la présence de spermatozoïdes macrocéphales pourrait aussi résulter de l'action de toxiques environnementaux tels que la fumée de cigarette [9] ou l'exposition à des agents mutagènes. Une augmentation de la fréquence des diploïdies et des disomies a été observée chez des patients traités par chimiothérapie [3] tandis qu'une exposition aux pesticides fait apparaître des hyperploïdies et des polyploïdies $[12,15]$. Ces molécules pourraient affecter l'appariement chromosomique ou la recombinaison génétique lors de la première division méiotique, ou la formation du fuseau de division lors des deux divisions de la méiose [11].

Dans les formes partielles, le pourcentage d'anomalies chromosomiques est supérieur au pourcentage de spermatozoïdes macrocéphales [1], ceci suggère que des spermatozoïdes de taille apparemment normale et susceptibles d'être utilisés en ICSI sont porteurs d'aneuploïdies ou même de diploïdies, avec les conséquences que l'on connaît sur le 
développement embryonnaire. Une autre information apparaît dans les résultats de la FISH. L'utilisation des sondes des chromosomes $X$ et $Y$ permet de distinguer les non-disjonctions de la première division méiotique $(X Y)$ de celles de la seconde ( $X X$ ou $Y Y$ ). Cette notion nous paraît intéressante car les mécanismes de ségrégation et donc de non-disjonction des chromosomes sont différents au cours des deux divisions méiotiques.

\section{CONSÉQUENCES SUR LA FONCTION DE REPRODUCTION ET CONDUITE PRATIQUE}

Les spermatozoïdes macrocéphales peuvent avoir plusieurs impacts sur la fonction de reproduction. Ils sont responsables d'infertilité car ils peuvent altérer le déroulement de toutes les étapes de la spermatogenèse, aboutissant à une diminution de la production de spermatozoïdes. Ils s'accompagnent d'anomalies de l'acrosome, d'une asthénozoospermie liée à la présence de plusieurs flagelles ou aux anomalies de l'axonème, et fréquemment d'une tératozoospermie totale.

Bien que Kahraman et coll. [10] aient pris en charge ces patients en procréation médicalement assistée, nous avons fait le choix d'exclure de tout programme d'assistance médicale à la procréation les couples dont l'homme avait un syndrome homogène. Cette attitude est justifiée par les taux d'anomalies chromosomiques retrouvées dans ces spermatozoïdes ainsi que par le taux élevé d'embryons à complément chromosomique anormal [10].

En ce qui concerne les formes partielles, nous avons montré que chez les patients infertiles dont au moins un spermogramme avait un taux égal ou supérieur à $20 \%$ de spermatozoïdes macrocéphales, on retrouvait des taux élevés d'anomalies chromosomiques [1]. La décision de prise en charge en assistance médicale à la procréation va dépendre du risque estimé d'échec de fécondation, d'arrêt de développement précoce de l'embryon et de déséquilibre chromosomique d'un embryon viable lié aux anomalies chromosomiques des spermatozoïdes.

Cependant, il faut noter qu'un déséquilibre chromosomique de l'embryon pourrait aussi résulter directement de la mutation responsable du syndrome dans le cas où son origine serait génétique. En effet, une mutation qui peut altérer à la fois les cycles cellulaires mitotiques et méiotiques pourrait conduire à une anomalie de ségrégation des chromosomes au cours du développement d'un embryon qui avait initialement un complément chromosomique normal.

Dans l'état actuel de nos connaissances, il est difficile de définir une conduite à tenir lorsque l'on dépiste la présence de spermatozoïdes macrocéphales à un taux modéré chez un patient infertile. En pratique avant toute AMP, nous proposons une étude des spermatozoïdes par FISH aux patients qui ont au moins $10 \%$ de spermatozoïdes macrocéphales sur au moins 2 spermogrammes. Les résultats sont alors discutés en commission multidisciplinaire. Le patient est ensuite informé des résultats.

Le principal risque étant la survenue d'anomalies chromosomiques de nombre, nous recommandons en cas de grossesse un suivi échographique et un dépistage sérique des anomalies chromosomique, associé si nécessaire à un calcul de risque intégré. Afin de ne pas compliquer le diagnostic prénatal en cas de risque augmenté, nous favorisons le transfert d'embryon unique. Notre étude nous aidera à définir partir de quel pourcentage de spermatozoïdes macrocéphales dans un éjaculat on peut considérer qu'il existe un risque, en particulier pour l'embryon, en sachant que le taux de spermatozoïdes macrocéphales peut varier d'un éjaculat à l'autre chez le même patient, et en particulier qu'il peut augmenter avec l'âge [4]. D'autre part, un échantillon d'ADN est conservé avec le consentement du patient pour permettre la recherche d'une cause génétique dans ces formes cliniques.

\section{CONCLUSION}

Les tératozoospermies monomorphes comme le syndrome des spermatozoïdes macrocéphales sont des pathologies rares parmi les patients pris en charge dans les centres d'assistance médicale à la procréation. L'origine génétique probable rend importante la recherche de cas familiaux et de consanguinité parmi les ascendants des patients porteurs.

D'un point de vue pratique, en dehors de la forme homogène du syndrome dans laquelle tous les spermatozoïdes ont un complément chromosomique anormal, l'ICSI peut être proposée. Le pronostic devra alors être évalué en fonction de chaque cas, et des examens complémentaires effectués, notamment en fonction des résultats de la FISH dans les formes partielles du syndrome. Une discussion multidisciplinaire d'évaluation des risques d'échec assistance médicale à la procréation, de fausse couche ou d'anomalies chromosomiques fœtales doit être engagée en fonction de chaque cas. Une information claire du couple est nécessaire avant toute prise en charge en assistance médicale à la procréation.

\section{REFERENCES}

1. ACHARD V., PAULMYER-LACROIX O., MERCIER G. et al. : Reproductive failure in patients with varoius percentages of macronuclear spermatozoa : high level of aneuploid and polyploid spermatozoa. J. Androl., 2007, 28 : 600-606.

2. BENZACKEN B., GAVELLE F.M., MARTIN-PONT B. et al. : Familial sperm polyploidy induced by genetic spermatogenesis failure : case report. Hum. Reprod., 2001, 16 : 2646-2651.

3. DE MAS P., DAUDIN M., VINCENT M.C. et al. : Increased aneuploidy in spermatozoa from testicular tumour patients after chemotherapy with cisplatin, etoposide and bleomycin. Hum. Reprod., 2001, 16 : 1204-1208.

4. DEVILLARD F., METZLER-GUILLEMAIN C., PELLETIER R. et al.: Polyploidy in large-headed sperm : FISH study of three cases. Hum. Reprod., 2002, 17 : 1292-1298.

5. DIETERICH K., SOTO RIFO R., FAURE A.K. et al. : Homozygous mutation of AURKC yields large-headed polyploid spermatozoa and causes male infertility. Nat. Genet., 2007, $39: 661-665$.

6. ESCALIER D. : Genetic approach to male meiotic division deficiency: the human macronuclear spermatozoa. Mol. Hum. Reprod., 2002, $8: 1-7$.

7. MITCHELL V., PEERG M-C., MARCHETTI C., et al.: Alterations morphologiques des spermatozoïdes en microsocopie électroniques : indications, phénotypes, fécondance et pronostic de fertilité. Andrologie, 2008: 18: 35-45.

8. GUTHAUSER B., VIALARD F., DAKOUANE M., IZARD V., ALBERT M., SELVA J. Chromosomal analysis of spermatozoa with normalsized heads in two infertile patients with macrocephalic sperm head syndrome. Fertil. Steril., 2006, 85 : 750.e5-750.e7.

9. HARKONEN K., VIITANEN T., LARSEN S.B., BONDE J.P., LAHDETIE $\mathrm{J}$. Aneupioidy in sperm and exposure to fungicides and lifestyle factors. ASCLEPIOS. A European Concerted Action on Occupational Hazards to Male Reproductive Capability. Environ. Mol. Mutagen, 1999, 34 : 39-46. 
10. KAHRAMAN S., AKARSU C., CENGIZ G. et al. : Fertility of ejaculated and testicular megalohead spermatozoa with intracytoplasmic sperm injection. Hum. Reprod., 1999, $14: 726-730$.

11. PADUNGTOD C., HASSOLD T.J., MILLIE E. et al. : Sperm aneuploidy among Chinese pesticide factory workers : scoring by the FISH method. Am. J. Ind. Med., 1999, 36 : 230-238.

12. RECIO R., ROBBINS W.A., BORJA-ABURTO V. et al. : Organophosphorous pesticide exposure increases the frequency of sperm sex null aneuploidy. Environ. Health Perspect., 2001, 109:12371240.

13. VICARI E., DE PALMA A., BURRELLO N. et al. : Absolute polymorphic teratozoospermia in patients with oligoasthenozoospermia is associated with an elevated sperm aneuploidy rate. J. Androl., 2003, 24 : 598-603.

14. VIVILLE S., MOLLARD R., BACH M.L., FALQUET C., GERLINGER P., WARTER S. : Do morphological anomalies reflect chromosomal aneuploidies ? : case report. Hum. Reprod., 2000, 15 : 2563-2566.

15. XIA Y., BIAN Q., XU L., CHENG S. et al. : Genotoxic effects on human spermatozoa among pesticide factory workers exposed to fenvalerate. Toxicology, 2004, 203 (1-3) : 49-60.

16. YUROV Y.B., SAIAS M.J., VORSANOVA S.G. et al. : Rapid chromosomal analysis of germ-line cells by FISH : an investigation of an infertile male with large-headed spermatozoa. Mol. Hum. Reprod., 1996, 2 : 665-668.

17. ZLOTOGORA J. : Germ line mosaicism. Hum. Genet., 1998, 102 : 381-386.

Manuscrit reçu : novembre 2007 ; accepté novembre 2007

\begin{abstract}
Macronuclear spermatozoa syndrome and its phenotypic variants: a form of teratozoospermia

Catherine METZLER-GUILLEMAIN, Vincent ACHARD, Georges MERCIER,Marie-Roberte GUICHAOUA
\end{abstract}

Macronuclear spermatozoa is a rare sperm defect, but always raises problems in the context of assisted reproductive technology (ART). The authors propose a review of the macronuclear spermatozoa syndrome and its phenotypic variants. In practice, ART can be proposed except in the homogeneous form of this sperm defect. Evaluation of chromosomal abnormality rates in the spermatozoa of these patients must be performed, followed by multidisciplinary discussion and clear information of the patients prior to ART.

Key words : macronuclear spermatozoa, teratozoospermia 